\title{
Local reinforcement effect of embedded strain gauges
}

\author{
A. Ajovalasit ${ }^{1, a}$, S. Fragapane ${ }^{1}$ and B. Zuccarello ${ }^{1}$ \\ ${ }^{1}$ Dipartimento di Meccanica, Università di Palermo, viale delle Scienze, 90128 Palermo, Italia
}

\section{Introduction}

The reinforcement effect of strain gauges installed on low Young's modulus materials has received attention by many researchers with respect to both strain gauges installed on the surface $[1,2]$ and embedded inside the material $[3,4]$.

In the case of strain gauges installed on the surface, the evaluation of the local reinforcement effect gives [5] the following correction coefficient $C$, i.e. the ratio between the actual strain $\varepsilon$ (without the strain gauge) and the strain $\varepsilon^{\prime}$ measured by the strain gauge:

$$
C=\frac{\varepsilon}{\varepsilon^{\prime}}=1+\frac{E_{s g}^{*}}{E_{s}}
$$

being

$$
E_{s g}^{*}=E_{s g} \frac{t_{s g}}{L_{s g}} \bar{\varphi}\left(\frac{L_{g}}{t_{s g}}, \frac{L_{s g}}{t_{s g}}\right)
$$

where $E_{s g}$ is the Young's modulus of the strain gauge, $E_{s g}^{*}$ is a characteristic of the strain gauge which gives the strain gauge sensitivity to the reinforcement effect (reduced Young's modulus of the strain gauge), $E_{s}$ is the Young's modulus of the specimen, $t_{s g}, L_{g}, L_{s g}$ are respectively thickness, total gauge length and grid length of the strain gauge, and $\bar{\varphi}$ is the mean value of a function $\varphi$ determined by a theoretical analysis [5]. This paper concerns the local reinforcement effect of embedded strain gauges that are frequently used especially inside plastic and composite materials.

\section{The embedded strain gauge}

In this paper the following assumptions used in the case of the strain gauge installed on the surface [5] are made, namely: (1) the specimen has the same width of the strain gauge, (2) the Young modulus and the thickness of the strain gauge is considered constant, that is independent from $x$ (Figure 1), (3) the distribution of the shear stresses at the interface between the strain gauge and the surrounding material is approximated by an exponential function.

It is then shown that, depending on the symmetry of the model shown in Figure 1 and neglecting the normal stresses acting on the strain gauge edges orthogonal to the $\mathrm{x}$-axis, the analysis of the embedded strain gauge is reduced to that of a strain gauge installed on the surface but having half

\footnotetext{
a e-mail : ajovalasit@dima.unipa.it
} 
thickness $\left(t_{s g} / 2\right)$ of the embedded gauge. The theoretical analysis of such equivalent model gives the correction coefficient $C_{e}$ of the embedded strain gauge:

$$
C_{e}=\frac{\varepsilon}{\varepsilon_{e}^{\prime}}=1+\frac{E_{s g}^{*_{e}}}{E_{s}}
$$

being

$$
E_{s g}^{* e}=\frac{1}{2} E_{s g} \frac{t_{s g}}{L_{s g}} \bar{\varphi}_{e}\left(\frac{L_{g}}{t_{s g}}, \frac{L_{s g}}{t_{s g}}\right)
$$

where the symbols have the same meaning as those contained in equations (1) and (2) and the subscript / superscript $e$ stands for embedded.

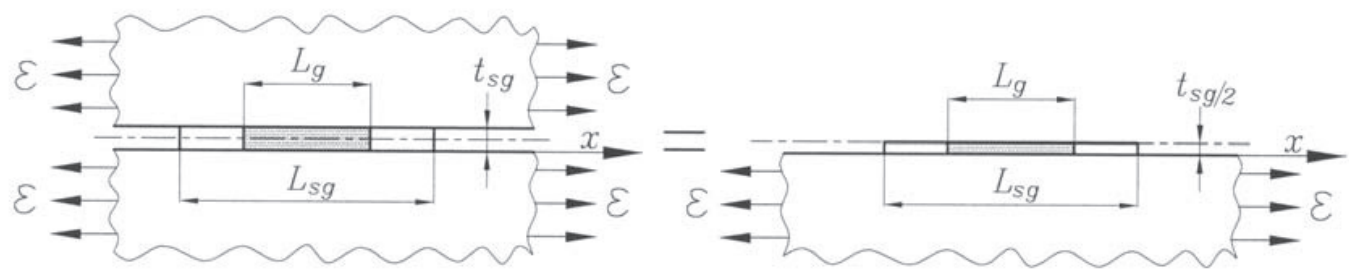

Figure 1 - Local reinforcement effect: equivalence between the embedded staring gauge (left) and the strain gauge installed on the surface (right)

The comparison between equations (4) and (2), gives the relationship between the sensitivities to the reinforcement effect, $E_{s g}^{* e}$ and $E_{s g}^{*}$, respectively of the embedded strain gauge and of the strain gauge installed on the surface.

$$
\frac{E_{s g}^{* e}}{E_{s g}^{*}}=\frac{C_{e}-1}{C-1}=\frac{1}{2} \frac{\bar{\varphi}_{e}}{\bar{\varphi}}
$$

Equation (5) shows that the local reinforcement effect of embedded strain gauges is smaller than the reinforcement effect of strain gauges installed on the surface. The magnitude of this reduction depends on the ratio $\bar{\varphi}_{e} / \bar{\varphi}$. In this paper the ratio $\bar{\varphi}_{e} / \bar{\varphi}$ was evaluated for some gauges (HBM and $\mathrm{MM})$ already considered in reference [5]. For these gauges the ratio $\bar{\varphi}_{e} / \bar{\varphi}$ is approximately 1 and thus the ratio $E_{s g}^{* e} / E_{s g}^{*}$ between the sensitivities to the reinforcement effect is about 0.5 .

The sensitivity to the reinforcement effect of an embedded strain gauge is thus approximately half of that of the same strain gauge installed on the surface, thus the local reinforcement effect of embedded strain gauges may be negligible in many practical applications. Experimental tests are currently underway to support the theoretical and numerical predictions.

\section{References}

1. Swam, J.W.: "Resistance strain gauges on thermoplastics". Strain 1973, 9(2), pp.56-59.

2. Little, E.G., Tocher, D., O’Donnell, P.: „, Strain gauge reinforcement of plastics“. Strain 1990, 26(3), pp. 91-98.

3. Dove R.C., Brasier, R.I., Baker, W.E., " Selection of gages for strain measurement at interior points”. Experimental Mechanics, 1962, 2(6), pp.189-190.

4. Bray, A., Desogus, S.: "Gli estensimetri annegati: nuovo metodo per l'analisi tridimensionale delle tensioni”. Ingegneria Meccanica, 1970, XIX (12), pp.29-35.

5. Ajovalasit, A., Zuccarello, B., "Local reinforcement effect of a strain gauge installation on low modulus materials", Journal of Strain Analysis, 40(7), 643-653, 2005. 